\title{
Effect of Communication Range with Node Cooperation in Wireless Passive Sensor Networks
}

\author{
${ }^{1}$ Farman Ullah, ${ }^{2}$ Aamir \\ Khan \\ ${ }^{1,2}$ COMSATS Insititute of IT \\ Quaid Avenue, Wah Cantt. \\ Pujnab, Pakistan
}

\author{
Imdad Ullah \\ SEECS, NUST, Islamabad, \\ Pakistan
}

\author{
Hasan Farooq \\ Universiti Tecknologi Petronas, \\ Malaysia
}

\begin{abstract}
Node cooperation is considered in this paper in the existence of two relaying protocols i.e. "Amplify and Forward (AF)" also called "Store and Forward (SF)" and the second relaying protocol is "Decode and Forward". The two stated protocols are different from each other and perform different operations on received data before retransmitting to next hop. Also the selection of each protocol is varying in accordance to the distance of a sensor node from the destination node (RF source). It is suggested in this literature that a relay node close to the source node will employ Decode and Forward and if it is close to the destination node, then Amplify and Forward will be employed. This work is achieved by first modelling an empirical system consists of single relay, source and destination. And the two relaying protocols (SF and DF) were modelled and implemented. This approach is then extended for three relay nodes and the two sets of relaying nodes were implemented again on every single node and then the output performances were compared. Finally using analytical approach, the communication range or the capacity of RF source to charge the sensor nodes in certain area was also evaluated and it is considered that the relay nodes within the prescribed range are successfully charged in charging stage. And in communication stage, the relay node was allowed to keep at various distances from the source node and evaluate the performance of each relaying protocol on a particular distance from source and destination.
\end{abstract}

\section{INTRODUCTION}

In wireless sensor networks, signal is severely degraded by the multipath signals which can be compensated with the use of diversity. Taking the advantageous of diversity, the destination node accumulates or combines all the received signals received via independent links and makes the final decision upon the transmitted bits. This characteristic of sensor networks is called the cooperative attempt and is always there when they are active. Sensor nodes, with this property enhance their quality of service and offer good BER compare to the conventional mode of communication. The senor nodes make use of their processing capabilities to locally carry out simple calculations and send out only interested data [5]. This approach or capability of WPSN can offer spatial diversity against fading in a wireless channel [22]-[23]. If there is no direct line of sight or having difficulty in communication, then one sensor node help another, called relay node in order to accomplish transmission from source to the desired destination [23]. This cooperative behaviour is adopted by each sensor node in the network along with the normal communication responsibility.
Node Cooperation results in various trades off in terms of resources e.g. data rate and transmit power. Because in cooperative mode if there are " $N$ " number of sensor nodes, then the total power would be divided into " $\mathrm{N}$ " number of portions and with that individual part of the total power, the data would be transmitted to each node. Hence, total power available at the single node divided and thus it is reduced for all users because of diversity [24].

The scenario that this work will consider would assume that all nodes work in the same band and therefore the source node will behave in broadcast manner, while the destination node is in multiple channel access mode as depicted in the following figure 1 . Channel between each pair of node is independent and have random effect on data.

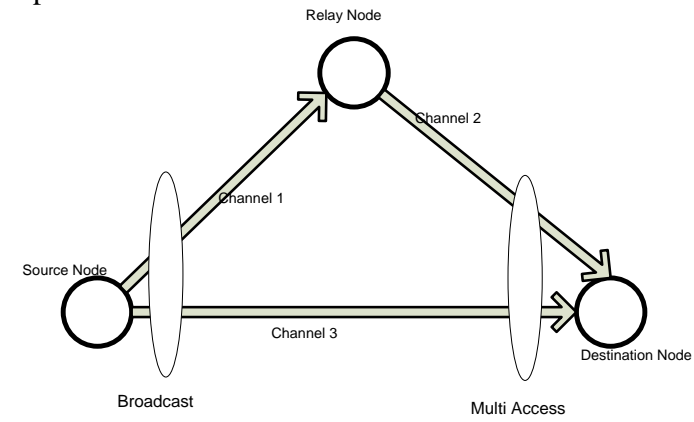

Figure 1: A 2 hops relaying system

\subsection{Relaying Protocols}

The two approaches are employed by the relaying nodes named as Amplify-and-Forward (AF) or Repeater and Decode-and-Forward (DF) in order to accomplish their job in the case when channel is severely fading. The need of two approaches arises when signal is very week and is unable to reach the destination node. The factors that weaken the signal are path loss (Large Scale Fading (LSF)) e.g. Reflection, Diffraction, Scattering etc. and Small Scale Fading (SSF) e.g. Rayleigh, Ricean, Nakagami, Doppler Spectrum, Autoregressive Model etc. [25]-[26] that could be resolved through the use of space, time and multiple antenna diversities. In a very simple case shown below is the 4 nodes system. In which two terminals $\mathrm{T} 1$ and $\mathrm{T} 2$ transmit to the destination terminals T3 and $\mathrm{T} 4$ respectively. But due to broadcast nature of wireless communication system, they also share resources with other nodes coming in the range of WPSN field [25] such as shown in Fig 3.2 and jointly communicate the data. 


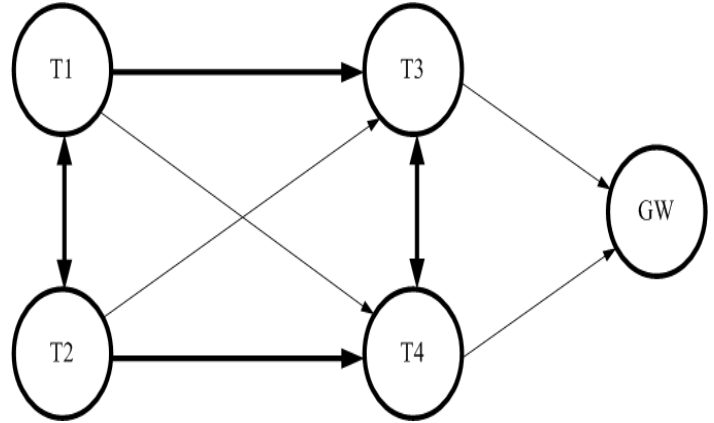

Figure 2: Relaying Nodes in Wireless Sensor Network

Each transmitting terminal focuses to send the data to their respective destination e.g. in a direct transmission between T1 and T3, the node T1 acts as source and T2 acts as relay node. So that in the case when fading is severe between $\mathrm{T} 1$ and $\mathrm{T} 3$ and transmission is not successful, then information is sent through the relay node (T2).

Relay transmission also happens when received information is severely corrupted that is called physically degraded relay channel e.g. the destination node receives data which is very different than the one received by relay node, and then the automatic retransmission occurs through the neighbour node. It is therefore, relay channel must be coping with the following three approaches [25],[27],[28].

$>$ Facilitation

- $\quad$ Relay should keep the interference smaller as much possible between the active communicating nodes to let them to transfer data more reliably.

- Relay must not help the source directly.

$>$ Cooperation

- Since resources are shared with T2 as well, therefore it fully decodes the source message and retransmits the source message in case $\mathrm{T} 1$ fails to deliver data successfully to destination node. Also in certain scenario, the received signal is not necessarily decoded, but simply reshape that signal to a higher amplitude and transmit towards the destination

$>$ Observation
- Relay encodes the quantized version of received signal and keeps record of every transmission that has been sent towards the destination.

In node cooperation, various protocols are employed to perform various operations. In fixed relaying, the received signal is either Amplify-and-Forward or Decode-andForward, depends upon the power availability.

Cooperative behaviour of sensor nodes created new approaches to enhance the sensor lifetime, low cost and multi functional operation in the sensor field. This approach created spatial diversity against fading in a wireless channel. In the case of sever fading channel, the source node is assisted by the relay channel to reach the information to the destination. This is basically done by the node relaying protocol called AF and DF. If the received signal SNR is below the threshold, due to which transmission is made unsuccessful, therefore message is encoded, re-encoded and retransmitted towards the destination. This decision is taken by the relay node on the single bit feedback, broadcasted by the destination that is at least reliably received by the relay node. Information is either corrupted by Reflection, Scattering or Diffraction etc or due to the Small Scale Fading effect of the channel.

\section{SYSTEM MODEL AND ACHIEVED RESULTS WITH DISCUSSION}

When signal is transmitted over a long communication path, the received signal is observed in a different form than the original. The envelope amplitude of this signal when observed would seem to be fluctuating as the time passes by. This effect is caused by "fading". This effect puts some limitations on the system design. However, if different signals are received, then fading over one path is different than another [34],[35]. Therefore if copy of the same signal is received via different links, then it is rarely happen that all channels are severely faded but it is good for reducing fading effect. This technique is called "diversity" which improves the combined signal to noise ratio and process of combining these signals at the receiver is called "Maximal Ratio Diversity" or Maximum Ration Combining (MRC) [36],[37].

\subsection{System Design}

Data passed through each relay node would be passed through the process shown by the following block diagram but only if the serving relay is choosing DF mode for retransmission of the received signal.

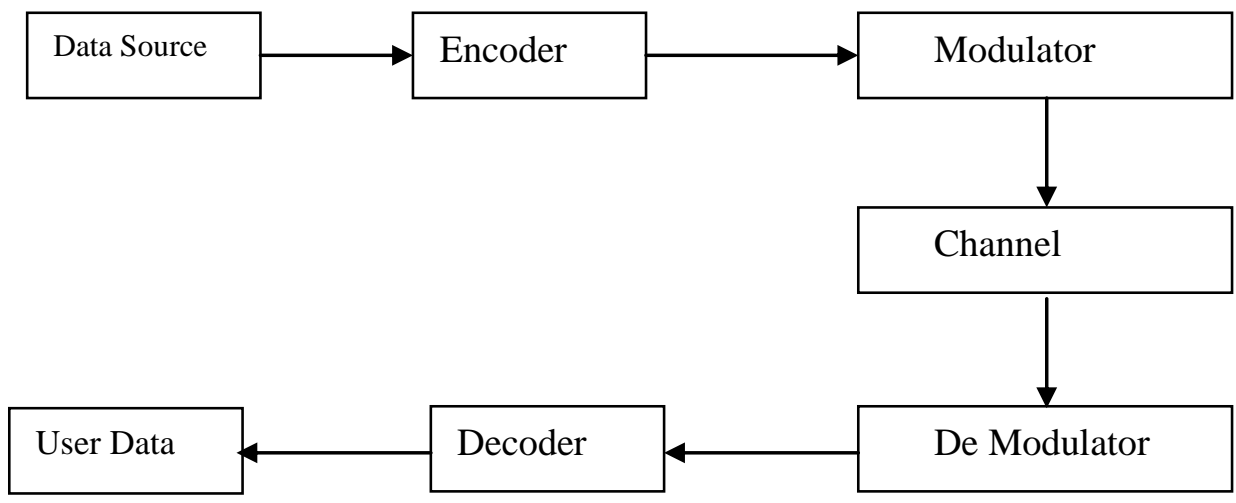

Figure 31: Block diagram of a Digital Communication Process System 
Block diagram shows that initially, the data source randomly generates bit stream of $0 \mathrm{~s}$ and 1s. Data stream is passed through encoder. Half convolutional encoder would be used in system design, which will introduce redundant bits equal to the information bits i.e. each information bit would have one redundant bit. The encoded bits would be modulated using different modulation schemes e.g. BPSK, QPSK etc. The modulator would generate a set of finite time duration waveforms and will provide mapping between the encoder output and the generated set of waveforms. The modulated signal is passed through flat fade channel and channel state information is known at the receiver. Channel introduces multiplicative noise but at the receiver, the received signal is also distorted by the AWGN noise. At the receiver, the same modulation scheme is used to de-map the signal and user data is decoded from the de-mapped signal.

The above block diagram would be integrated in each relay which could be more generally seen in the form of following flow chart.

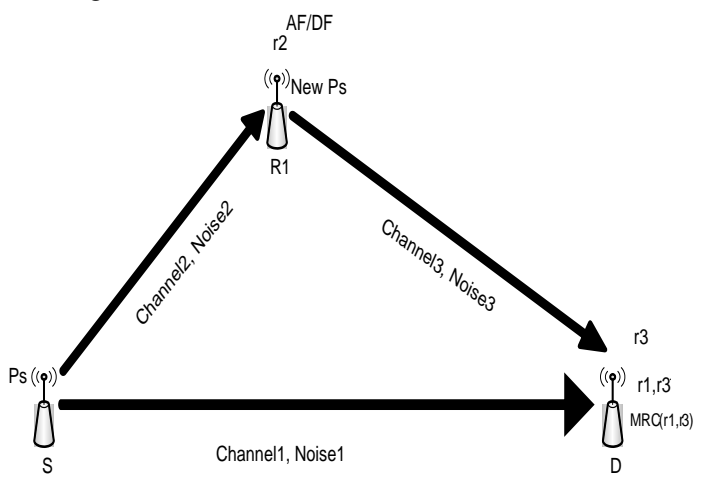

Figure 4: One Relaying node using either AF or DF

\subsection{Communication Coverage of the RF source}

This section will calculate range or communication coverage of RF source. So the sensor nodes coming within the calculated range would be successfully charged.

In order to do this, the battery free sensor node (P2110) is considered. P2110 converts the received RF energy into DC, which is stored in capacitor. The capacitor is constantly charged unless the charging threshold is obtained. Soon as the threshold level achieved, the output voltage is enabled and device starts functioning. Similarly when charging level drops below the threshold level, the output voltage is turned off [21]. Typical voltage on sensor node is $1.24 \mathrm{~V}$ according to [31]. Based on this value, power received by the RF source could be worked out by recalling equation of received power.

$$
P_{r}=\frac{\left|V_{t}\right|^{2}}{8\left(R_{t}+R_{L}\right)}
$$

$\mathrm{P} 2110$ can best perform in the range of $902-928 \mathrm{MHz}$ (typically $915 \mathrm{MHz}$ ) with RF and sensor node impedances of 50 ohms.

Substituting

$V_{t}=1.24 V R_{r}=50 \Omega R_{l}=50 \Omega$ in equation 1

$P_{r}=\frac{\left|V_{t}\right|^{2}}{8\left(R_{r}+R_{l}\right)}=\frac{(1.24)^{2}}{8(50+50)}$

$P_{r}=1.92 \mathrm{~mW}$

$P_{r}(d B)=-27 d B W$

$P_{r}(d B)=2.8 d B m$

$\{X d B W+30=X d B m\}$
The calculated $\mathrm{P}_{\mathrm{r}}$ is the minimum power by which the sensor could be activated. This power would at least cross the threshold level of the sensor and would let the sensor to start functioning.

Typical EIRP used by the RF source is $4 \mathrm{~W}$ to power the sensor node with $6 \mathrm{dBi}$ transmit antenna gain. The received antenna gain is proposed as $4 \mathrm{dBi}[40]$.

$$
\begin{gathered}
P_{t}=4 \mathrm{~W} \Rightarrow P_{t}(d B)=6.02 d B W \Rightarrow P_{t}(d B)=36.02 \mathrm{dBm} \\
G_{r}=4 d B i \\
G_{t}=6 d B i \\
\left.\lambda=\frac{3 * 10^{8} \mathrm{~m} / \mathrm{s}}{915 \mathrm{MHz}}=0.323 \mathrm{~m} \ldots \ldots \ldots \ldots \ldots . . . . . . . . . .3\right)
\end{gathered}
$$

Substituting the above parameters in Friis transmission equation

$$
\begin{gathered}
P_{r}=P_{t}\left(\frac{\lambda}{4 \pi R_{r f}}\right)^{2} G_{t} G_{r} \\
P_{r}=P_{t}+G_{t}+G_{r}+20 \log \left(\frac{\lambda}{4 \pi R_{t, r}}\right) \\
2.8=36.02+6+4+20 \log \left(\frac{0.323}{4 * 3.14 * R_{t, r}}\right) \\
R_{t, r}=\frac{1}{\frac{4 * 3.14 * 10}{0.323}} \\
R_{t, r}=3.72 m
\end{gathered}
$$

Equation 4 shows that, the sensors within the radius of $3.72 \mathrm{~m}$ would be successfully charged. Also it can be said that the sensor node would only have $2.8 \mathrm{dBm}$ power to utilize and to distribute among the neighbour sensor nodes over the radius of $3.72 \mathrm{~m}$.

This range could be increased or decreased i.e. it could be set to the desired value by taking one of the following resistor values.

Received voltage at the sensor node could also be increased or decreased by connecting the resistor of values according to $R=\frac{249 k\left(V_{\text {out }}-1.195\right)}{3.32-V_{\text {out }}}$ and $R=\frac{297.47 \mathrm{~K}}{V_{\text {out }}-3.32}$

Now by keeping source node at a fixed position i.e. $3.72 \mathrm{~m}$ and let this node to transmit data with power what it can have from the RF source at this point. The relay node performance could be demonstrated at various distances with respect to the fixed source node as could be seen from figure 5 .

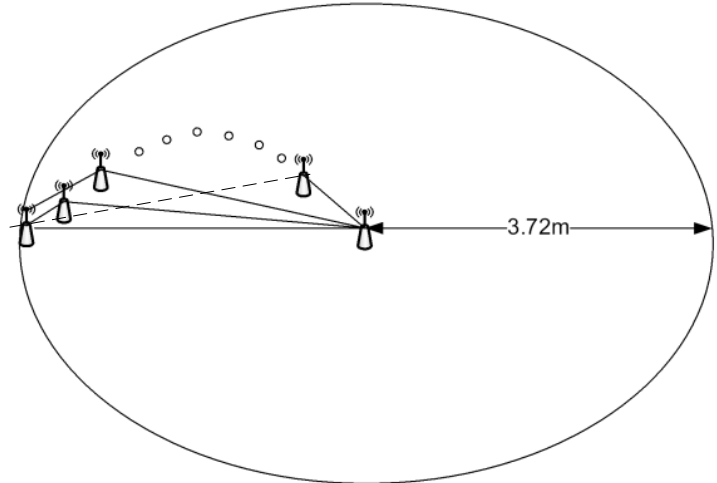

Figure 5. Relay output performance with respect to various distances 
In above figure, the relay node is kept at various distances in order to see the performances. In this case, it could be said (in simplest form) that as sensor node approach towards the RF source, the sensor node receives more and more power in the same unit of time, because for the same charging time duration and equal size plate of the inside capacitor would be more quickly charged at distance close to the RF source compare to the one kept farther from the RF source.

The source node transmits with constant power and as the relay node retransmits the received data with always larger power than the source power. Following distances were fixed for this experiment and received power is evaluated at each new distance as shown in the following table. Received power is evaluated using Friis Transmission equation.

\begin{tabular}{|l|l|l|}
\hline S.No & Distance & $\begin{array}{l}\text { Relay's Received } \\
\text { Power }\end{array}$ \\
\hline 1 & 3.3067 & 5.8393 \\
\hline 2 & 3.2550 & 5.9914 \\
\hline 3 & 3.1886 & 6.1829 \\
\hline 4 & 3.1000 & 6.4316 \\
\hline 5 & 2.9760 & 6.7684 \\
\hline 6 & 2.7900 & 7.2527 \\
\hline 7 & 1.8600 & 9.4616 \\
\hline 8 & 0.9300 & 11.7186 \\
\hline 9 & 0.6200 & 12.6426 \\
\hline 10 & 0.4650 & 13.1963 \\
\hline 11 & 0.3720 & 13.5819 \\
\hline 12 & 0.3100 & 13.8734 \\
\hline
\end{tabular}

Table 1: Received power by the sensor node at various distances

It is obvious from table 1 , as the sensor node approaching towards the destination node, the received power at each point is constantly increasing. With this power, the sensor node will retransmit the received data. Each time the relay node retransmits the received data with higher power as approaching towards the destination node, the results of which could be seen from figure 6.

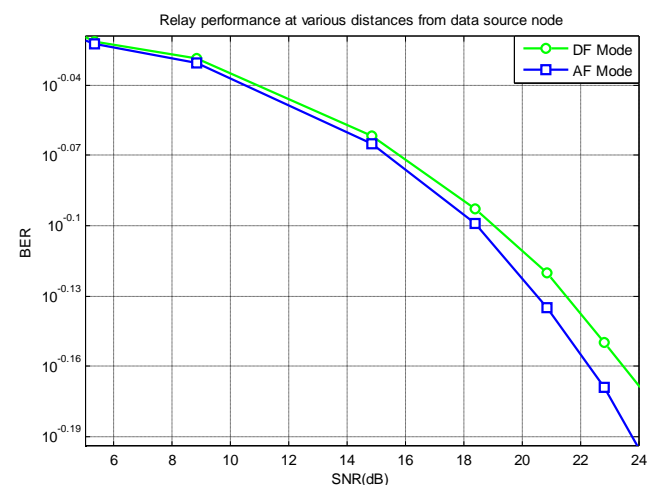

Figure 6 Relay outputs at various received power (obtained at various distances) with respect to the source node.

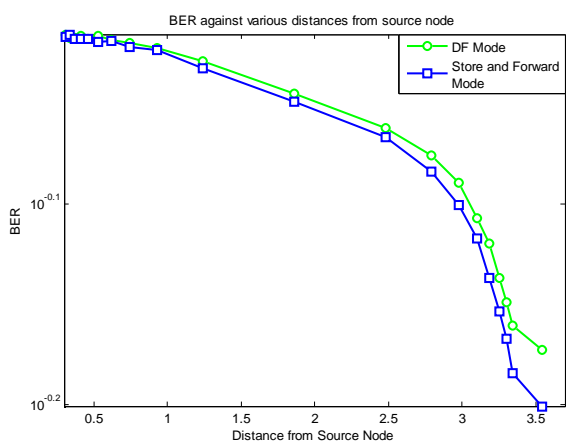

Figure7. Relay outputs at various distances with respect to the data source node

\section{CONCLUSION}

It has been considered that relay is fixed in the middle of source and destination link and plot BER against the SNR. At this point, when source node transmits the generated data with half the available power, the use of two relaying protocols proves that $\mathrm{AF}$ gives good performance compared to $\mathrm{DF}$ and Conventional mode of transmissions. As a result of the outputs, it could be added that if the received data is demodulated from carrier and decoded to get the original bits from the received data, then there is high chance that the final message would be highly modified and would be deviated from the source message.

\section{REFERENCES}

[1] Jennifer Yick, Biswanath Mukherjee, Dipak Ghosal, "Wireless Sensor Network Survey," J.Yick et al./ Computer Networks 52 (2008), pp.2292-2330, April 2008.

[2] X,-Y. Li; "Multicast Capacity of Wireless Ad Hoc Networks", IEEE/ACM Transaction on Networking, vol.7, no.13, pp.950-961, June2009.

[3] G,-K. Chang, Z. Jia, J. Yu. A. Chowdhury, T. Wang, G. Ellinas, "Super-Broadband Optical Wireless Access Technologies," OFC/NFOEC 2008.

[4] A. Bereketli and O. B. Akan, "Communication Coverage in Wireless Passive Sensor Networks," IEEE Comm. Letters, vol. 13, no. 2, pp. 133-135, Feb. 2009.

[5] I.F.Akyildiz, W.Su, Y.Sankarasubramaniam, E. Cayirci, "Wireless Sensor Networks: A Survey "Computer Networks 38 (2002), pp.393-422, Dec 2001.

[6] Alexander Becher, Zinaida Benenson, and Maximillian Dornseif, "Tampering with Motes: Real-World Physical Attacks on Wireless Sensor Networks," pp. 1-15.

[7] Adrian Perrig, John Stankovic, David Wagner, "Security in wireless sensor networks," Commun. ACM, 47(6), pp.53-57, 2004.

[8] Elaine Shi and Adrian Perrig, "Designing secure sensor networks," IEEE Wireless Communications, 11(6), pp.38-43, December 2004.

[9] O. B. Akan, M. T. Isik, B. Baykal, "Wireless Passive Sensor Networks," IEEE Comm. Magazine, vol. 47, no. 8, pp. 92-99, Aug. 2009. 
[10] D. Sakamoto and H. Higaki "Wireless Multihop Transmission with Buffering in Neighbour Sensor Nodes for Short Delay," IEEE Communications Society, WCNC 2009.

[11] Shi Yongjie, Sun Guiling, Liu Bo. Li Weixiang, "Design of Wireless Sensor Network Node Based on C8051F020 and CC1100 Fiber Bragg Grating," IEEE, pp. 1-4.July 2010.

[12] C. Intanagonwiwat, R. Govindan, and D. Estrin, "Directed Diffusion: A Scalable and Robus Communication Paradigm for Sensor Networks," Proc. ACM MobiCom 'OO, Boston, MA, 2000, pp. 56-57.

[13] H. Stockman, "Communication by means of Reflected Power,” Proc. I.R.E., vol. 36, 1948, pp.1196-1204.

[14] M. Kossel, H. R. Benedickter, R. Peter, Batchtold, "Microwave Backscatter Modulation Systems," in IEEE MIT-S Digest 2000, pp.1427-1430.

[15] C.Turner, "Backscatter modulation of Impedance Modulated RFID tags," pp. 1-5, Feb 2003.

[16] M. Talha Isik Ozgur B. Akan, "Padre: Modulated Backscattering-based Passive Data Retrieval in Wireless Sensor Networks," IEEE Comm So, WCN 2009 Proc,.

[17] F. Kocer, P.M. Walsh, and M. P. Flynn. "Wireless Remotely Powered Telemetry in $0.25 \mathrm{~m}$ CMOS," Proc, IEEE RFIC, June 2004, pp. 339-342.

[18] Yanqiu Li, Hongyun Yu, Bo Su, and Yonghong Shang, "Hybrid Micropower Source for Wireless Sensor Network," IEEE Sensors Journal, Vol 8, N0.6, June 2008, pp. 678-681.

[19] A. K. Marath, Y. W. M. Chia, C. C. Ko, "Performance Analysis of a Homodyne Receiver in Modulated Backscattered System for Intelligent Transportation." IEEE 1999, pp. 1198-1202.

[20] K. Han, Y. Choi, S. Choi, and Y. Kwon, "Power Amplifier Characteristic aware energy efficient Transmission Strategy," LNCS, vol.4479, pp. 37-48, Springer, Nov.2007.

[21] Product Datasheet "P2110-915MHz RF Powerharvester Receiver," PowerCast, Rev A-2010/04, pp.1-12.

[22] M. R. Souryal, B. R. Vojcic, "Performance of Amplifyand-Forward and Decode-and-Forward Relaying in Rayleigh Fading with turbo codes,"IEEE ICASSP, pp.IV68-IV-684, 2006

[23] J. Lee, S. Kim, H. S. T. Kwon, Y. Choi, J. S and A. Par, "Downlink Node Cooperation with Node Selection Diversity," IEEE, 2005

[24] N. Laneman, D. N. C. Tse, G. W. Wornell, "Cooperative Diversity in Wireless Networks Efficient Protocols and Outage Behavior," IEEE Trans: on infor theory, vol,50, No, 12, pp. 2062-3080, 12 Dec 2004.

[25] A. Nosratinia, T. E. Hunter, A. Hedayat, "Cooperative Communication Wireless Networks," Adaptive Antennas and Mimo Systems for Wireless Comm, IEEE Comm: Magazine, pp. 74-80, Oct 2004.

[26] N. Laneman, D. N. C. Tse, G. W. Wornell, "Cooperative Diversity in Wireless Networks Efficient Protocols and Outage Behavior," IEEE Trans: on infor theory, vol,50, No, 12, pp. 2062-3080, 12 Dec 2004.
[27] J. G. Proakis, Digital Communication, $4^{\text {th }}$ ed. New York: Mc Graw-Hill, Inc., 2001.

[28] T. M. Cover and A. A. El Gamal, "Capacity theorems for the relay channel," IEEE Trans. Infor, Theory, vol. IT-25, pp.572-584, Sept.1979.

[29] M. Yu and J. Li, "Is amplify-and-forward practically better than decode-and-forward or vice versa?", Proc. IEEE Int. Conf. Acoustics, Speech, and Signal Processing (ICASSP). Vol. 3, pp.365-368. March 2005.

[30] M-S. Alouini, A. Goldsmith, "Capacity of Rayleigh Fading Channels Under Different Adaptive Transmission and Diversity-Combining Techniques," IEEE Trans: on Vehicular Tech:, Vol:48, No. 4, pp. 1165-1181, July 1999.

[31] T. Sunaga, S. Sampei, "Performance of multi-level QAM with post-detection maximal ration combining space diversity of digital land-mobile radio communications," IEEE Trans: Vehicular Technology, Vol. 42, pp.294-301, Aug 1993

[32] J. N. Laneman, "Cooperative Diversity in Wireless Networks: Algorithms and Architectures," Ph.D. dissertation, Massachusetts Institute of Technology, Cambridge, MA, Aug. 2002

[33] X. Bao, J. LI, "Efficient Message Relaying for Wireless User Cooperation: Decode-Amplify-Forward (DAF) and Hybrid DAF and Coded-Cooperation," IEEE Trans: on Wireless Comm:, Vol. 6, pp. 3975-3985, Nov. 2007.

[34] T. Q. Duong, H-J. Zepernick, "Hybrid Decode-AmplifyForward Cooperative Communications with Multiple Relays," IEEE Comm: Soc: WCN 2009 Proc:, pp. 1-6

[35] C. R. C. M. Da Silva, M.D Yacoub, "A Generalized Solution for Diversity Combining Techniques in Fading Channels," IEEE Trans: on Microwave Theory and Techniques, Vol. 50, No.1, pp. 46-50, Jan 2002.

[36] B.S. Paul, R. Bhattacharjee, "Maximal ratio combining of two amplify-forward relay branches with individual 1 inks experiencing Nakagami fading," IEEE, pp.1-4, 2007.

[37] A. Sendonaris, E. Erkip, B. Aazhang, "User Cooperation diversity-part-1: system description," IEEE Transactions on Comm: Vol. 51, no.11, Nov 2003.

[38] A. Sendonaris, E. Erkip, B. Aazhang, "User Cooperation diversity-part-II: Implementation aspects and performance analysis," IEEE Transactions on Comm: vol.51, no. 11, Nov 2003

[39] Product Datasheet "P2110-915MHz RF Powerharvester Receiver," PowerCast, Rev A-2010/04, pp.1-12.

[40] http://www.rfwirelesssensors.com/2010/04/powercastp2110-battery-free-wireless-sensor-node-2010-04-30/

[41] G. Hoblos, M.Staroswiecki, and A.Aitouche, "Optimal Design of Fault Tolerant Sensor Networks," IEEE Int'l. Conf. Cont. Apps., Anchorage AK. Sept. 2000,PP.467-72.

[42] Bulusu et al., "Scalable Coordination for Wireless Sensor Networks: Self Configuring Localization Systems," ISCTA 2001, Ambleside, U.K, July 2001 